\title{
Selective increases of bifidobacteria in gut microflora improve high-fat-diet-induced diabetes in mice through a mechanism associated with endotoxaemia
}

\author{
P. D. Cani • A. M. Neyrinck • F. Fava • C. Knauf • \\ R. G. Burcelin • K. M. Tuohy • G. R. Gibson • \\ N. M. Delzenne
}

Received: 22 May 2007 / Accepted: 6 July 2007 / Published online: 6 September 2007

(C) Springer-Verlag 2007

\begin{abstract}
Aims/hypothesis Recent evidence suggests that a particular gut microbial community may favour occurrence of the metabolic diseases. Recently, we reported that high-fat (HF) feeding was associated with higher endotoxaemia and lower Bifidobacterium species (spp.) caecal content in mice. We therefore tested whether restoration of the quantity of caecal Bifidobacterium spp. could modulate metabolic endotoxaemia, the inflammatory tone and the development of diabetes.

Methods Since bifidobacteria have been reported to reduce intestinal endotoxin levels and improve mucosal barrier function, we specifically increased the gut bifidobacterial content of HF-diet-fed mice through the use of a prebiotic (oligofructose [OFS]).

Results Compared with normal chow-fed control mice, HF feeding significantly reduced intestinal Gram-negative and
\end{abstract}

Electronic supplementary material The online version of this article (doi:10.1007/s00125-007-0791-0) contains supplementary material, which is available to authorised users.

P. D. Cani $(\bowtie) \cdot$ A. M. Neyrinck $\cdot$ N. M. Delzenne $(\bowtie)$

Unit of Pharmacokinetics, Metabolism, Nutrition and Toxicology,

Université catholique de Louvain,

Av. E. Mounier, 73/69,

1200 Brussels, Belgium

e-mail: patrice.cani@uclouvain.be

e-mail: nathalie.delzenne@uclouvain.be

F. Fava $\cdot$ K. M. Tuohy $\cdot$ G. R. Gibson

Food Microbial Sciences Unit, Department of Food Biosciences,

The University of Reading,

Reading, UK

C. Knauf $\cdot$ R. G. Burcelin

Rangueil Institute of Molecular Medicine, I2MR, IFR31,

Toulouse, France
Gram-positive bacteria including levels of bifidobacteria, a dominant member of the intestinal microbiota, which is seen as physiologically positive. As expected, HF-OFS-fed mice had totally restored quantities of bifidobacteria. HFfeeding significantly increased endotoxaemia, which was normalised to control levels in HF-OFS-treated mice. Multiple-correlation analyses showed that endotoxaemia significantly and negatively correlated with Bifidobacterium spp., but no relationship was seen between endotoxaemia and any other bacterial group. Finally, in HF-OFS-treated-mice, Bifidobacterium spp. significantly and positively correlated with improved glucose tolerance, glucose-induced insulin secretion and normalised inflammatory tone (decreased endotoxaemia, plasma and adipose tissue proinflammatory cytokines).

Conclusions/interpretation Together, these findings suggest that the gut microbiota contribute towards the pathophysiological regulation of endotoxaemia and set the tone of inflammation for occurrence of diabetes and/or obesity. Thus, it would be useful to develop specific strategies for modifying gut microbiota in favour of bifidobacteria to prevent the deleterious effect of HF-diet-induced metabolic diseases.

Keywords Bifidobacteria · Diabetes · Endotoxin · GLP-1 · Glucagon-like peptide-1 · Gut microflora - Inflammation · Obesity - Prebiotics

$\begin{array}{ll}\text { Abbreviations } \\ \text { HF } & \text { high-fat } \\ \text { HF-Cell } & \text { high-fat cellulose } \\ \text { HF-OFS } & \text { high-fat oligofructose } \\ \text { LPS } & \text { lipopolysaccharide } \\ \text { MIB } & \text { mouse intestinal bacteria } \\ \text { OFS } & \text { oligofructose } \\ \text { spp. } & \text { species }\end{array}$




\section{Introduction}

Evidence suggests that obesity and metabolic disorders (type 2 diabetes and insulin resistance) are tightly linked to inflammation $[1,2]$. Although obesity results from interactions between genetic and environmental factors, eating habits contributing to an increase in fat intake can promote metabolic diseases.

In models of diet-induced and genetic obesity, adipose tissue has increased production and content of pro-inflammatory cytokines such as IL-1, IL-6 and TNF- $\alpha$ [3, 4].

Cytokines have been shown to cause insulin resistance $[5,6]$, which favours hyperinsulinaemia and excessive hepatic and adipose tissue lipid storage. However, while extensive research has been dedicated to the effects of inflammatory reactions on energy metabolism, the triggering factor linking inflammation to a high-fat (HF)-dietinduced metabolic syndrome remains to be determined.

An innovative hypothesis was recently proposed: the gut flora could be an important factor affecting energy disposal and could be implicated in metabolic disease associated with obesity [7-11]. One observation reported that young adult germ-free mice had $40 \%$ less body fat than their conventionalised counterparts on the same diet. Similarly, lean axenic mice colonised with microbiota from $o b / o b$ mice increased body weight [7]. The authors suggested that gut microbiota from obese mice allowed energy to be salvaged from otherwise non-digestible dietary polysaccharides producing substantial elevations in serum glucose and insulin, both factors that trigger lipogenesis. However, this hypothesis of microbiota-induced lipogenesis did not explain the differential effect on body weight of a HF diet vs regular chow, since axenic mice fed a HF diet did not gain weight. This suggests that a bacterially related factor was responsible for HF-diet-induced obesity [9] and that lipids alone were not sufficient to promote obesity and inflammation.

The relationship between HF feeding and the development of a low-grade inflammatory tone is unclear, but may be related to the microbiota present in the digestive tract. With regard to the role of gut microbiota in the development of metabolic diseases, we recently reported [12] that HF feeding in mice induced a low-grade inflammatory tone and that metabolic disease was associated with reduced numbers of Bifidobacterium species (spp.) in caecal content and higher plasma endotoxin (lipopolysaccharide [LPS]) concentrations (defined as metabolic endotoxaemia). Hence, LPS appears to be a molecular link between HF feeding, microbiota and inflammation. LPS was identified as a novel factor triggering the onset of HF diet-induced obesity and type 2 diabetes [12]. We have also shown that mice lacking the major LPS co-receptor CD14 were resistant to HF-diet-induced inflammation and metabolic diseases [12]. Since bifidobacteria reduced intestinal endotoxin levels and improved mucosal barrier function [13-15], we decided to specifically increase the gut bifidobacterial content of HF-diet-treated mice through prebiotic dietary fibre (oligofructose [OFS]), which can reproducibly modulate the gut microbiota typified by increased bifidobacteria [16].

We therefore tested the hypothesis that the pattern of bacterial changes occurring through dietary intervention with OFS may control the occurrence of metabolic diseases in mice fed a HF diet for 14 weeks.

\section{Methods}

Animals Male C57bl6/J mice (12 weeks old; Charles River, Brussels, Belgium) were housed in a controlled environment (12 h daylight cycle, lights off at 18:00 hours) with free access to food and water. All mouse experiments were approved by the local ethics committee and housing conditions were as specified by the Belgian Law of 14 November 1993 on the protection of laboratory animals (agreement number LA 1230314). Mice were killed by cervical dislocation after a $5 \mathrm{~h}$ period of fasting. Caecum (full and empty) and adipose tissues (epididymal, subcutaneous and visceral) were precisely dissected, weighed, immersed in liquid nitrogen and stored at $-80^{\circ} \mathrm{C}$, for further analysis.

Diet and experimental groups Mice ( $n=8$ per group) were fed four different experimental diets for 14 weeks as follows: (1) standard diet (control) (A04; UAR, Villemoisson-sur-Orge, France) [17]; (2) HF diet containing 49.5\% fat $(\mathrm{g} / 100 \mathrm{~g}$ of total dry diet) corresponding to $72 \%$ of the total energy content (UAR, Epinay-sur-Orge, France); (3) a mix of HF diet and a fermentable dietary fibre (OFS) (HFOFS) (kind gift from Orafti, Tienen, Belgium) [18]; and (4) a mix of HF diet and a non-fermentable dietary fibre (microcrystalline cellulose [HF-Cell]) (Vivapur microcrystalline cellulose; J. Rettenmaier \& Söhne, Weissenborn, Germany). Dietary fibre was added in a proportion of 90:10 (weight of HF diet:weight of fibre). Nutritional compositions of the experimental diets are provided in the Electronic supplementary material (ESM Table 1).

Glucose tolerance test Oral glucose tolerance tests were performed after 13 weeks of treatment in mice that had been fasted for $6 \mathrm{~h}$. Glucose was orally administered (3 g/ $\mathrm{kg}$ body weight, $660 \mathrm{~g} / 1$ glucose solution) and blood glucose determined through a glucose meter (Roche Diagnostics, Meylan, France) using $3.5 \mu \mathrm{l}$ of blood collected from the tip of the tail vein before and at administration of glucose load (-30 and $0 \mathrm{~min})$ and after glucose load $(15,30,60,90$, 
$120 \mathrm{~min}$ ). To assess plasma insulin concentration, $20 \mu \mathrm{l}$ of blood was sampled $30 \mathrm{~min}$ before and $15 \mathrm{~min}$ following the glucose load.

Biochemical analyses Plasma LPS determinations were performed using a kit based on a Limulus amoebocyte extract (LAL kit; Cambrex BioScience, Walkersville, MD, USA); samples were diluted 1:50 and heated for $10 \mathrm{~min}$ at $70^{\circ} \mathrm{C}$. Plasma insulin concentration was determined in $5 \mu \mathrm{l}$ of plasma using an ELISA kit (Mercodia, Uppsala, Sweden) according to the manufacturer's instructions. Cytokines were determined in $12 \mu \mathrm{l}$ of plasma using a kit (Bio-Plex Multiplex; Bio-Rad, Nazareth, Belgium) and measured using Luminex technology (Bio-Plex; Bio-Rad).

Microbial quantification in intestinal contents by fluorescence in situ hybridisation (FISH) analysis Caecal contents collected post mortem from mice were stored at $-70^{\circ} \mathrm{C}$. Samples were thawed on ice and diluted 1:10 in sterile ice cold PBS ( $0.1 \mathrm{~mol} / \mathrm{l}$ phosphate, $\mathrm{pH} 7.0)(0.1 \mathrm{~mol} / \mathrm{l}$ phosphate, $\mathrm{pH}$ 7.0). The suspension was homogenised by pipetting and centrifuging at $3,500 \times \mathrm{g}$ for $15 \mathrm{~min}$ to remove particulate matter. The supernatant fraction containing bacterial cells was fixed overnight in $4 \%(w / v)$ paraformaldehyde. The bacterial cells were then washed and re-suspended twice in sterile PBS and finally stored in $500 \mathrm{ml}$ ethanol/1 PBS at $-20^{\circ} \mathrm{C}$ until hybridisation with appropriate molecular probes targeting specific regions of 16S rRNA. The probes used were: EREC 482 [19] for Eubacterium rectaleClostridium coccoides group, Lab158 [20] for lactobacilli and enterococci and Bif164 [21] for Bifidobacterium spp. (Gram-positive bacteria); and Bac303 [22] for BacteroidesPrevotella spp., MIB 661 [23] for Bacteroides mouse intestinal bacteria (MIB), SRB 687 [24] for sulphatereducing bacteria and probe D [25] for Enterobacteriaceae spp. (Gram-negative bacteria) within the murine intestinal microflora. The nucleic acid stain DAPI was used for total bacterial counts. The DNA probes were tagged with the Cy3 fluorescence dye so that the hybridised samples could be examined using fluorescence microscopy. Results were expressed as $\log _{10}$ (bacterial cells per g caecal content wet weight).

Real-time quantitative PCR Total RNAs from white visceral adipose tissue were prepared using a reagent (TriPure; Roche, Basel, Switzerland) as described [26]. PCRs were performed using a sequence detection system instrument and software (AbiPrism 5700; Applied Biosystems, Foster City, CA, USA) as described [26]. Primer sequences for the targeted mouse genes are available upon request (patrice. cani@uclouvain.be).

Statistical analysis Results are presented as mean \pm SEM. Statistical significance of differences was analysed by one- way ANOVA followed by post hoc (Bonferroni's multiple comparison test). Correlations between parameters where assessed by Pearson's correlation test, using GraphPad Prism version 4.00 for Windows (GraphPad Software, San Diego, CA, USA). A value of $p<0.05$ was regarded as statistically significant.

\section{Results}

Specific increase of bifidobacteria by prebiotics The maintenance of mice on a HF diet resulted in a significant modulation of population levels of the dominant members of the intestinal microbiota as compared with control diet (Fig. 1). The major modifications observed were a dramatic reduction in quantities of bifidobacteria in HF-fed mice compared with control and the Gram-negative Bacteroides MIB, as previously described [12]. To determine the metabolic role of bifidobacteria following HF-diet feeding, we took advantage of the prebiotic model, known to specifically increase gut bifidobacterial contents. For 14 weeks mice were fed a HF diet enriched or not with prebiotic non-digestible carbohydrate (OFS) (HF-OFS) or non-prebiotic non-digestible carbohydrate (HF-Cell) as negative control. Importantly, quantities of bifidobacteria in HF-OFS fed mice were restored and even higher than those of control mice (Fig. 1a), confirming the role of OFS as a prebiotic. Numbers of the newly recognised Gramnegative operating taxonomic unit, MIB, which reside within the Cytophaga-Flavobacter-Bacteroides phylum (referred to here as Bacteroides MIB), were markedly reduced in all HF-diet-treated mice. These bacteria are closely related to the Bacteroides-Prevotella group, but not quantified by the probe Bac303. Together with the $E$. rectale- $C$. coccoides group, they constitute the dominant members of the mouse intestinal microbiota [23]. Numbers of the $E$. rectale $-C$. coccoides group were also significantly lower in animals fed the HF diet compared with control and even more so in HF-Cell mice (Fig. 1).

Prebiotics lower endotoxaemia We observed that HF and HF-Cell mice showed a significant increase of endotoxaemia whereas HF-OFS normalised to control values (Table 1). This suggests that the increase in bifidobacteria by prebiotics protected against endotoxaemia induced by a HF diet.

Gram-positive Bifidobacterium spp. negatively correlated with endotoxaemia To identify whether one specific group of gut bacteria was involved in the determination of endotoxaemia, we performed multiple correlations analyses between the major Gram-positive and Gram-negative bacteria present in the caecal contents of mice (Fig. 2). 
Fig. 1 Modulation of gut bacteria by dietary treatments. Caecal bacterial content of a Bifidobacterium spp., b Eubacterium rectale-Clostridium coccoides group, c lactobacillienterococci, d Bacteroides spp., e Enterobacteriaceae, f Bacteroides MIB, g sulphate-reducing bacteria and $\mathbf{h}$ total bacteria. Bacterial quantities are expressed as $\log _{10}$ (bacterial cells/g caecal content wet weight). Data are mean $\pm \mathrm{SE}$. Data with different superscript letters are significantly different $p<0.05$, according to the post hoc ANOVA statistical analysis. $C T$ Control diet, $H F$ high-fat diet, $H F-C e l l$ high-fat diet and non-fermentable dietary fibre, $H F$-OFS high-fat diet and fermentable dietary fibre
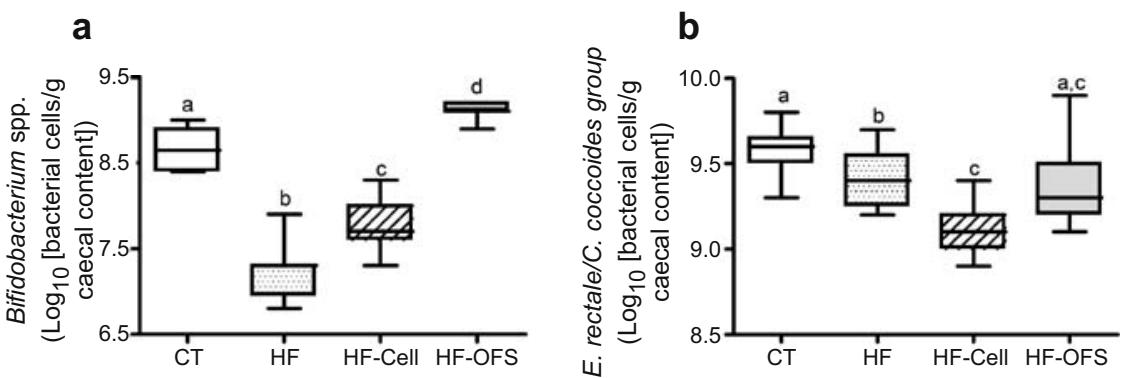

C

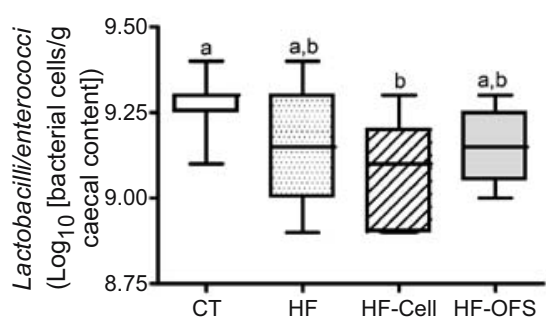

d

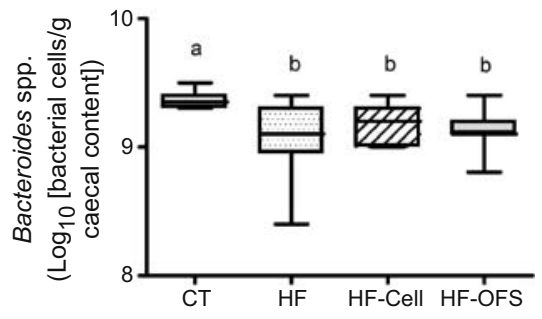

f

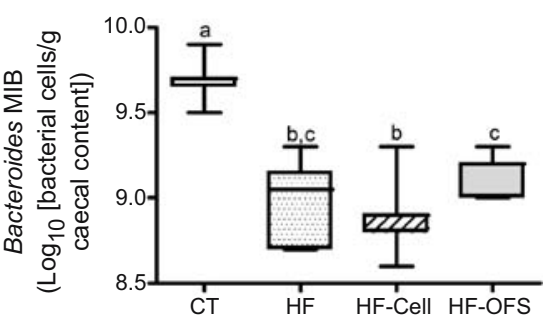

h

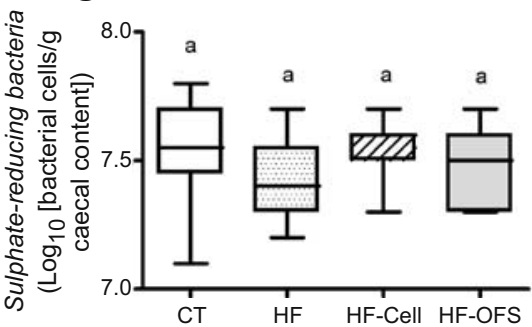

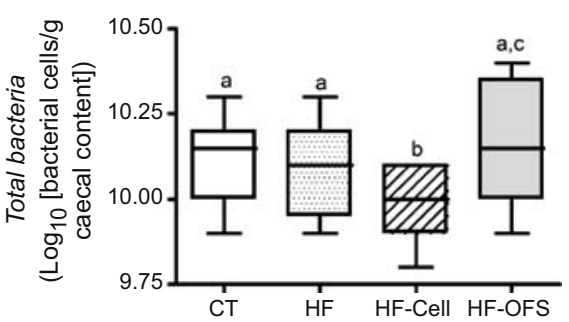

Endotoxaemia significantly and negatively correlated with Bifidobacterium spp. (Fig. 2a), but no relationship was seen between endotoxaemia and any other bacterial group (Fig. 2). Since we found that prebiotic fed mice only exhibited higher gut bifidobacteria and normalised endotoxaemia, we studied the putative causative link between such parameters and metabolic disorders in these mice.

Prebiotics improve glucose tolerance and restore glucoseinduced insulin secretion HF mice showed strong glucose intolerance, as shown by glucose excursion and AUC data (Fig. 3a,b). HF-OFS improved glucose tolerance compared with HF and HF-Cell mice (Fig. 3a,b). Fasted insulinaemia was significantly increased in $\mathrm{HF}$ and $\mathrm{HF}-\mathrm{Cell}$ mice compared with control (Fig. 3c) and insulin secretion following glucose load was almost absent in HF and HFCell mice (Fig. 3c,d). In contrast, HF-OFS mice showed normal fasting plasma insulin levels and restored glucoseinduced insulin secretion (Fig. 3c,d). Moreover, fasted insulin and glycaemic response were positively correlated with plasma endotoxin levels (Fig. 4a,b) and negatively correlated with bifidobacteria (Fig. 4c,d). This set of data suggests that the specific increase of bifidobacteria and subsequent lower level of endotoxaemia measured in HFOFS mice impacted on glucose homeostasis.

Prebiotic supplementation improves body weight gain and energy intake, reduces fat mass development and increases colonic glucagon-like peptide-1 precursor $\mathrm{HF}$ diet increased total body weight gain and fat mass by 2.5 -fold 
Table 1 Body weight, plasma, adipose tissue inflammatory markers and GLP-1 precursor mRNA

\begin{tabular}{|c|c|c|c|c|}
\hline Parameters & CT & $\mathrm{HF}$ & HF-Cell & HF-OFS \\
\hline Body weight gain (g) & $4.82 \pm 0.52^{\mathrm{a}}$ & $11.56 \pm 1.34^{\mathrm{b}}$ & $8.03 \pm 0.52^{\mathrm{c}}$ & $7.74 \pm 0.57^{\mathrm{c}}$ \\
\hline Daily energy intake (kJ/mouse) & $37.82 \pm 2.46^{\mathrm{a}}$ & $79.04 \pm 6.22^{\mathrm{b}}$ & $64.03 \pm 1.29^{\mathrm{b}, *}$ & $61.02 \pm 1.21^{\mathrm{b}, *}$ \\
\hline \multicolumn{5}{|c|}{ Adipose tissue weight ( $\mathrm{g} / 100 \mathrm{~g}$ body weight) } \\
\hline Visceral & $0.59 \pm 0.05^{\mathrm{a}}$ & $1.16 \pm 0.11^{\mathrm{b}}$ & $0.89 \pm 0.03^{\mathrm{a}}$ & $0.90 \pm 0.05^{\mathrm{a}}$ \\
\hline Epididymal & $1.38 \pm 0.1^{\mathrm{a}}$ & $4.74 \pm 0.54^{\mathrm{b}}$ & $3.21 \pm 0.26^{\mathrm{c}}$ & $3.16 \pm 0.22^{\mathrm{c}}$ \\
\hline Subcutaneous & $1.42 \pm 0.08^{\mathrm{a}}$ & $5.28 \pm 0.65^{\mathrm{b}}$ & $3.85 \pm 0.47^{\mathrm{c}}$ & $3.18 \pm 0.14^{\mathrm{c}}$ \\
\hline Plasma endotoxin (EU/ml) & $5.02 \pm 0.56^{\mathrm{a}}$ & $9.03 \pm 1.25^{\mathrm{b}}$ & $11.28 \pm 3.52^{\mathrm{b}}$ & $6.16 \pm 0.47^{\mathrm{a}}$ \\
\hline \multicolumn{5}{|l|}{ Visceral adipose tissue mRNA (AU) } \\
\hline$I l-1$ & $3.59 \pm 0.68^{\mathrm{a}}$ & $5.54 \pm 2.24^{\mathrm{a}}$ & $5.88 \pm 1.57^{\mathrm{a}}$ & $2.33 \pm 0.69^{\mathrm{a}, * *}$ \\
\hline Pai-1 & $3.98 \pm 0.83^{\mathrm{a}}$ & $17.44 \pm 3.74^{\mathrm{b}}$ & $65.89 \pm 25.79^{\mathrm{c}}$ & $11.58 \pm 4.92^{\mathrm{a}, \mathrm{b}}$ \\
\hline $\operatorname{Tnf}-\alpha$ & $2.88 \pm 0.63^{\mathrm{a}}$ & $4.63 \pm 1.67^{\mathrm{a}}$ & $4.40 \pm 1.21^{\mathrm{a}}$ & $1.97 \pm 0.67^{\mathrm{a}}$ \\
\hline Colon proglucagon mRNA (AU) & $1.49 \pm 0.55^{\mathrm{a}}$ & $1.87 \pm 0.43^{\mathrm{a}}$ & $2.78 \pm 0.20^{\mathrm{a}}$ & $6.96 \pm 0.95^{\mathrm{b}}$ \\
\hline
\end{tabular}

Data are mean \pm SEM

$A U$ Arbitrary units

${ }^{*} p=0.1$ vs $\mathrm{HF}$

${ }^{* * *} p=0.06$ vs HF-Cell

a,b,c Data with different superscript letters are significantly different $p<0.05$, according to the post hoc ANOVA statistical analysis.

$C T$ Control diet, $H F$ high-fat diet, $H F$-Cell high-fat diet and non-fermentable dietary fibre, HF-OFS high-fat diet and fermentable dietary fibre

and 3- to 4-fold, respectively, compared with the standard diet. HF-OFS and HF-Cell-fed mice had reduced energy intake compared with HF (Table 1). In addition, treated mice showed significantly reduced body weight gain and fat mass development compared with HF. Nevertheless, HF-OFS fed mice had 20\% lower subcutaneous adipose tissue than HF-Cell counterparts (Table 1). Here again we found that body weight and visceral fat mass correlated positively with plasma endotoxin levels (Fig. 5a,b) and negatively with bifidobacteria (Fig. 5c,d). We have previously described how OFS feeding promotes endogenous glucagon-like peptide-1 (GLP-1) production (i.e. colonic proglucagon mRNA) $[17,18,27-29]$. We therefore measured colonic proglucagon mRNA in the present study. HF-OFS fed mice exhibited a strong increase of proglucagon mRNA precursor (by about 4.6-, 3.7- and 2.5-fold, compared with control, HF and HF-Cell respectively) (Table 1).
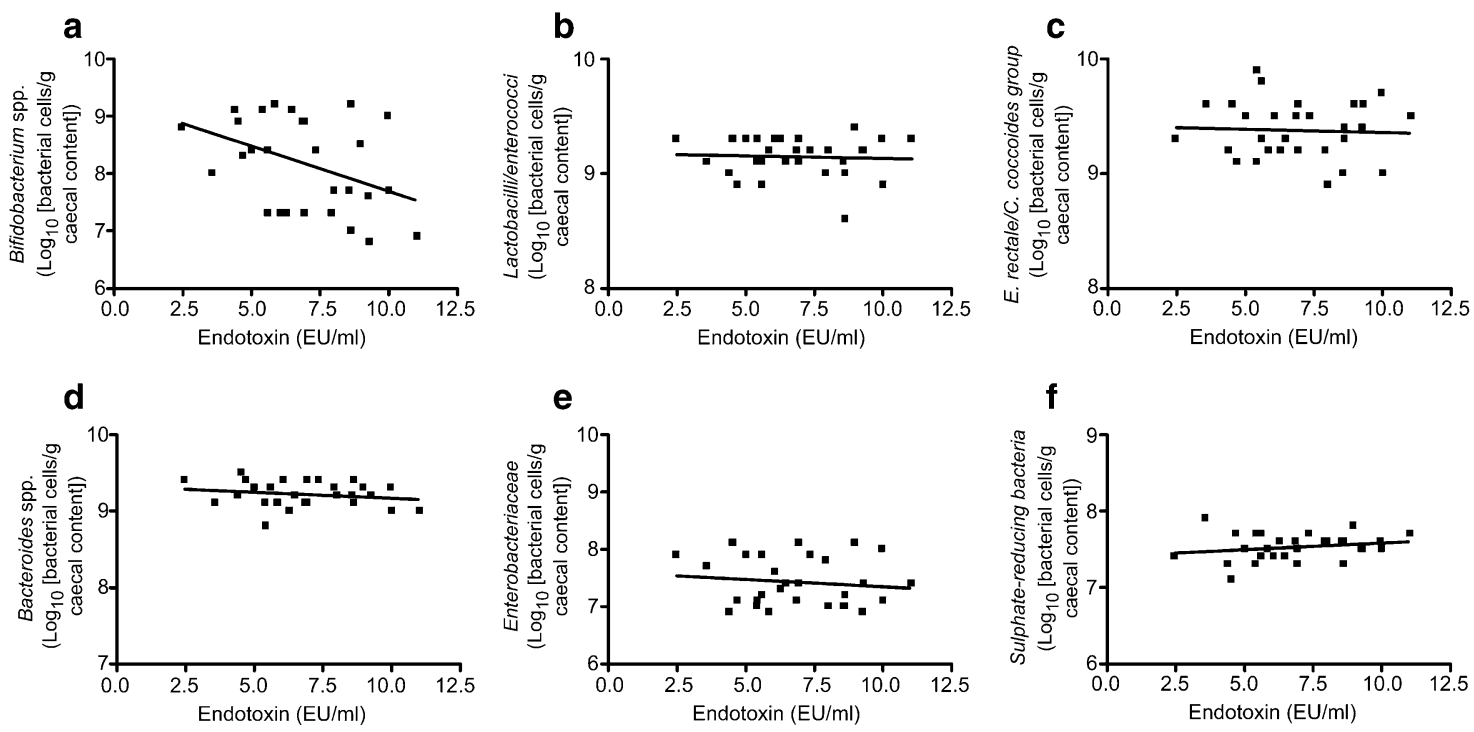

Fig. 2 Gram-positive Bifidobacterium spp. negatively correlates with endotoxaemia. Correlations between groups of bacteria present in the caecal content of mice. Main Gram-positive gut bacteria: a Bifidobacterium spp., b lactobacilli-enterococci, c Eubacterium rectaleClostridium coccoides group. Main Gram-negative gut bacteria: d Bacteroides spp., e Enterobacteriaceae, f sulphate-reducing bacteria. Bacterial quantities and plasma endotoxin levels are expressed as indicated. Pearson's $r$ correlations: $r=-0.41, p=0.025$ (a); $r=-0.06$, $p=0.79$ (b); $r=-0.03, p=0.81$ (c); $r=-0.18, p=0.3$ (d); $r=-0.12, p=$ $0.51(\mathbf{e}) ; r=0.21, p=0.28$ (f) 
a

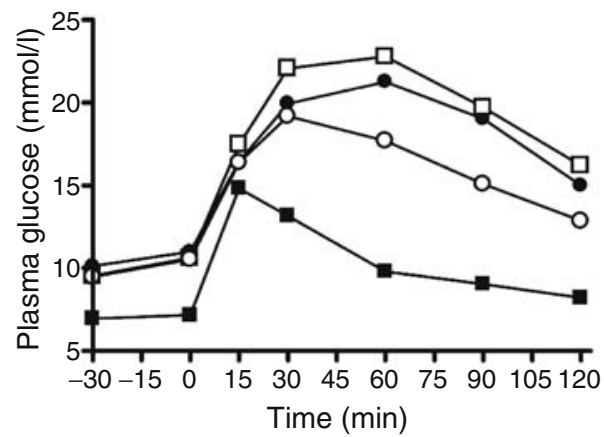

C

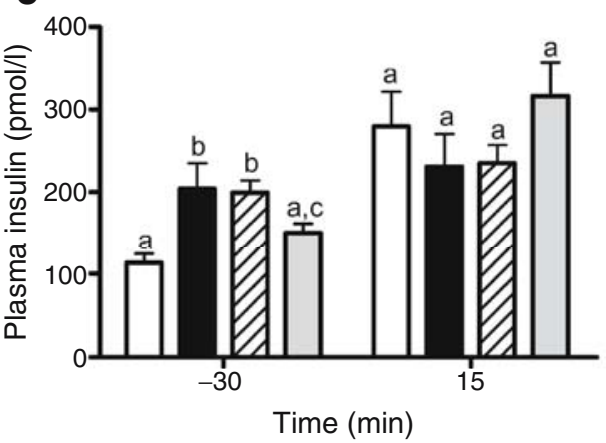

Fig. 3 Prebiotics improve glucose tolerance and glucose-induced insulin secretion. a Plasma glucose ( $\mathrm{mmol} / \mathrm{l})$ following an oral glucose load $(3 \mathrm{~g} / \mathrm{kg})$ in control mice fed a normal chow diet (closed squares) or in mice fed an HF diet (closed circles) or an HF-OFS diet (open circles) or an HF-Cell diet (open squares) for 14 weeks. b AUCs in the same groups. c Plasma insulin concentrations $30 \mathrm{~min}$ before $(-30)$ and $15 \mathrm{~min}$ after (15) an oral glucose load in control mice fed a normal chow diet (open bars) or in mice on HF diet (closed bars) or on an

Prebiotics control high-fat-diet-induced inflammation Since we observed higher endotoxaemia in HF-fed and HF-Cellfed mice, we measured plasma IL- $1 \alpha$, IL-1 $\beta$ and IL-6. It was seen that IL- $1 \alpha$, IL- $1 \beta$ and IL- 6 were increased in HF mice compared with control (Fig. 6). HF-OFS had significantly normalised IL- $1 \alpha$ and IL- 6 cytokines plasma levels compared with HF and tended to have decreased IL$1 \beta$, with HF-Cell mice showing intermediary levels. The adipose tissue mRNA concentrations of Il-1 (also known as Il1), TNF- $\alpha$ (also known as Tnf) and plasminogen activator inhibitor type 1 (Pai-1, also known as Serpinel) were increased in HF and HF-Cell-fed mice, whereas this increase was blunted in HF-OFS fed mice (Table 1). These last results demonstrate that the lower fat mass and body weight observed in HF-Cell mice is not a prerequisite for reduction of the inflammatory tone as measured in the plasma and adipose tissue of HF-OFS fed mice.

Here, too, we found that plasma cytokines were positively correlated with plasma endotoxin levels (Fig. 7a-c) and negatively correlated with bifidobacteria (Fig. $7 \mathrm{~d}-\mathrm{f}$ ).
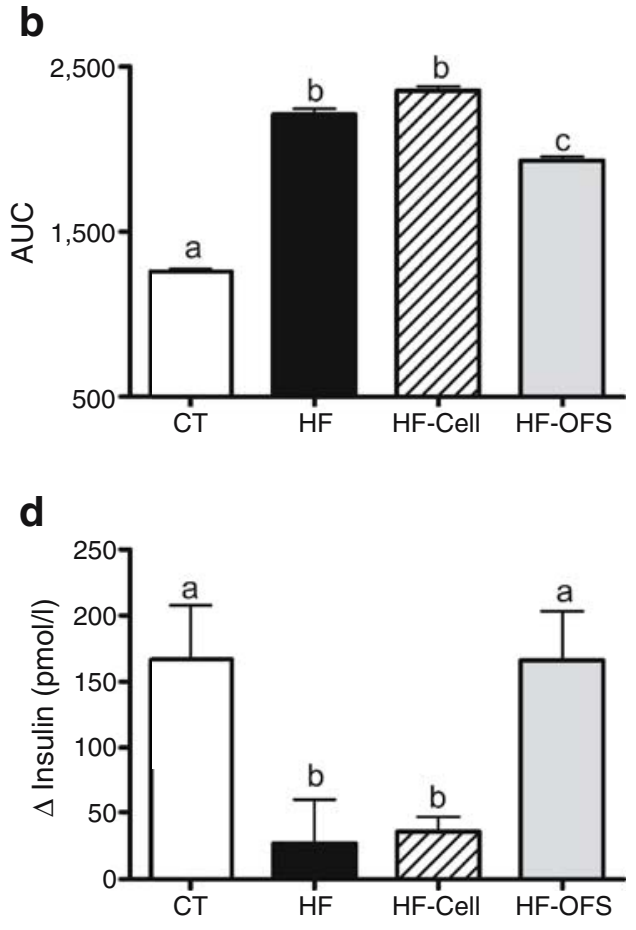

HF-Cell diet (hatched bars) or an HF-OFS diet (grey bars) for 14 weeks. $\mathbf{d} \Delta$ Insulin between $30 \mathrm{~min}$ before and $15 \mathrm{~min}$ after an oral glucose load. Data are mean \pm SE. Data with different superscript letters are significantly different $p<0.05$, according to the post hoc ANOVA statistical analysis. $C T$ Control diet, $H F$ high-fat diet, $H F$ Cell high-fat diet and non-fermentable dietary fibre, $H F-O F S$ high-fat diet and fermentable dietary fibre

\section{Discussion}

Recent studies have highlighted key mammalian host-gut microbial relationships, suggesting that the gut microbiota play an important role in energy metabolism [7]. Moreover, our recent studies have demonstrated that HF diet-fed mice developed insulin resistance and inflammation by a mechanism directly dependent on a gut bacterial Gramnegative derived compound, namely LPS [12]. Here we report that HF feeding alters the intestinal microbiota composition: quantities of the dominant Gram-positive groups Bifidobacterium spp. and E. rectale-C. coccoides group were reduced, as were the numbers of the numerically dominant murine Gram-negative group, Bacteroides MIB, compared with controls fed a standard diet. Moreover, we found that among the different gut bacteria analysed, plasma LPS concentrations correlated negatively with Bifidobacterium spp. Since, bifidobacteria have been shown to reduce intestinal endotoxin levels and improve mucosal barrier function [13-15], we decided to specifically increase the gut bifidobacterial content of HF-diet-treated mice 
Fig. 4 Glucose tolerance and fasted plasma insulin correlate positively with endotoxaemia and negatively with

Bifidobacterium spp. Correlation between endotoxaemia and a glycaemic response to glucose load (AUC) or b fasted insulin.

c Correlation between

Bifidobacterium spp. and glycaemic response to glucose load (AUC) or $\mathbf{d}$ fasted insulin. Pearson's $r$ correlations: $r=0.36$, $p=0.05$ (a); $r=0.41, p=0.027$ (b); $r=-0.51, p=0.005$ (c); $r=-0.46, p=0.011$ (d) a
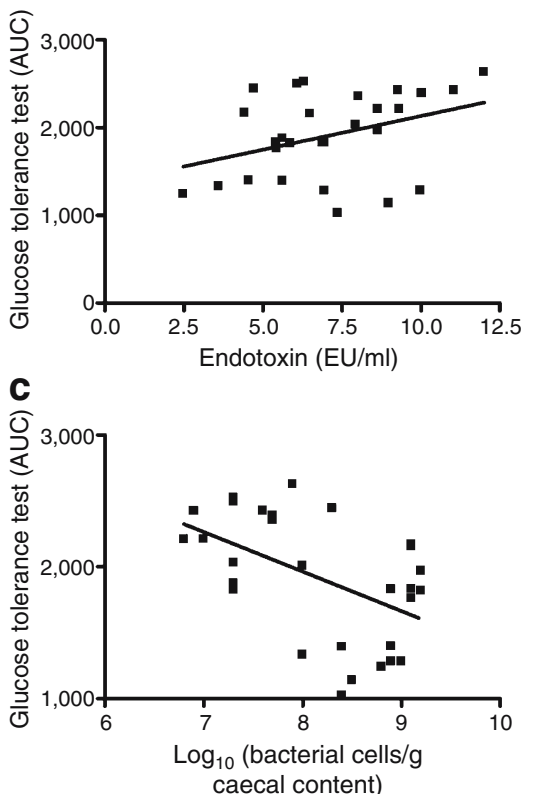

b

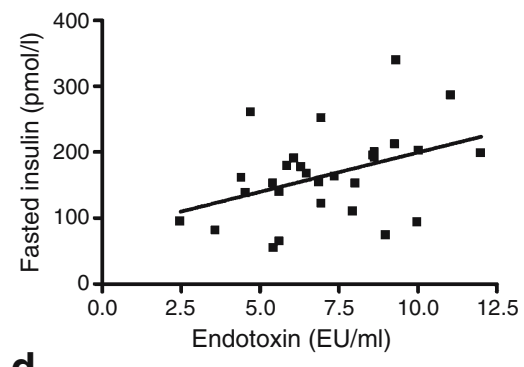

d

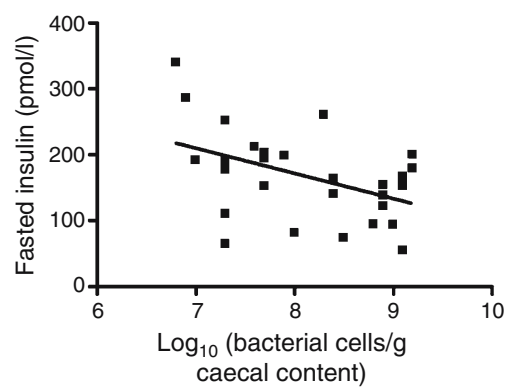

through supplementation with prebiotic dietary fibre (OFS). Mice fed the prebiotic dietary fibre had normal endotoxaemia. This observation strongly correlated with improved glucose tolerance as explained by the normalisation of glucose-induced insulin secretion. Although glucose-induced insulin secretion was normalised in HF-OFS mice, glucose tolerance following a glucose load was improved, but did not reach control values. This suggests that OFSfed mice remained insulin resistant. According to these results, we have previously shown that HF-diet-induced hepatic insulin resistance was normalised in OFS fed mice, without significant improvement in whole-body insulin sensitivity [18]. Moreover, the improved hepatic insulin sensitivity observed in HF-OFS fed mice was strongly associated with a significantly lower hepatic inflammatory tone [18].

Since we observed higher endotoxaemia in HF-diet-fed mice, we measured plasma and adipose tissue inflammatory
Fig. 5 Body weight and fat mass correlate positively with endotoxaemia and negatively with Bifidobacterium spp. Correlations between endotoxaemia and $\mathbf{a}$ body weight or $\mathbf{b}$ adipose fat mass. c Correlations between Bifidobacterium spp. and body weight or $\mathbf{d}$ adipose fat mass. Pearson's $r$ correlations: $r=0.61$, $p=0.005$ (a); $r=0.39, p=0.03$ (b); $r=-0.61, p=0.0003$ (c); $r=$ $-0.46, p=0.01$ (d) a
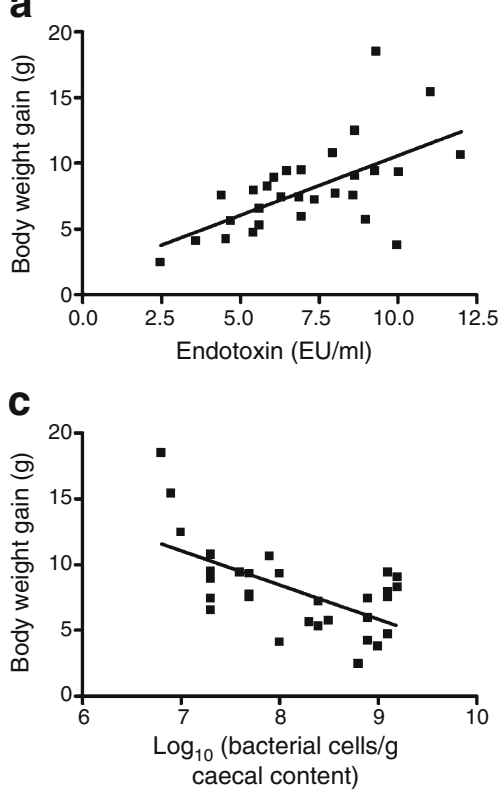

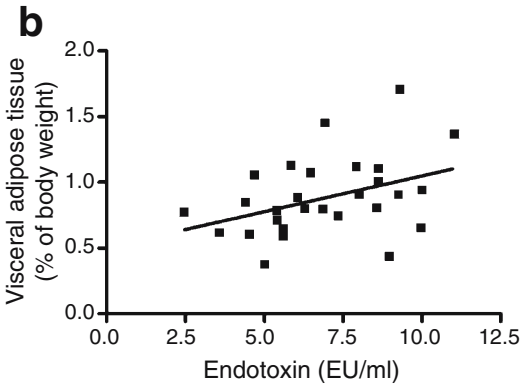

d

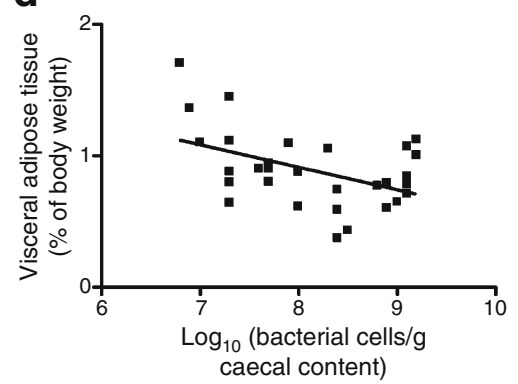




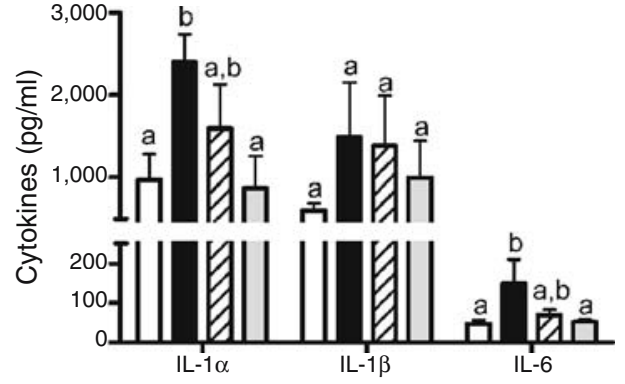

Fig. 6 Prebiotics lower plasma pro-inflammatory cytokines. Plasma cytokine concentrations $(\mathrm{pg} / \mathrm{ml})$ in mice fed normal chow diet (open bars), HF diet (closed bars), HF-Cell diet (hatched bars) or HF-OFS diet (grey bars) for 14 weeks. Data are mean \pm SE. Data with different superscript letters are significantly different $p<0.05$, according to the post hoc ANOVA statistical analysis

markers. We found that HF-diet feeding increased plasma IL- $1 \alpha$, IL- $1 \beta$ and IL- 6 concentrations, but this increase was prevented only in prebiotic treated mice. It seems that bifidobacteria and endotoxaemia are therefore linked to metabolic disorders. In line with our results, Brun et al. have recently demonstrated that obese and diabetic mice $(o b / o b$ and $d b / d b)$ exhibited significantly higher plasma endotoxin levels, which correlated to higher plasma inflammatory markers [30]. Multiple Pearson's correlations analysis of our data revealed that endotoxaemia positively correlated with glucose intolerance, fasted insulinaemia, inflammatory markers, adipose tissue and body weight gain, while bifidobacteria negatively correlated with these parameters. Collectively, our data indicate that HF-dietinduced metabolic disorders could be modulated through a number of related aspects.

We have shown that increased endotoxaemia observed during HF feeding could be one outcome of the modulation of gut microbiota. Moreover, we cannot exclude that intestinal mucosa permeability could be increased following HF feeding and restored through prebiotic treatment. The exact mechanism by which prebiotic fibre lowers endotoxaemia and systemic inflammatory tone is poorly understood. It is recognised that bifidobacteria are the main gut bacteria involved in the positive effects observed after prebiotic supplementation. Indeed, bifidobacterial supplementation has been associated with lower bacterial translocation and endotoxaemia, leading to a decrease of the inflammatory cascade activation in several models of gut bacteria translocation [13-15, 31]. Similarly, other studies have reported that probiotics and gut microbiota modulation through prebiotic ingestion may improve or prevent disruption of intestinal epithelial barrier function and consequently reduce intestinal permeability in rodents and humans $[32,33]$. Other studies strongly suggest that the products of OFS fermentation such as short-chain fatty acids (butyrate, propionate and lactate) positively influence

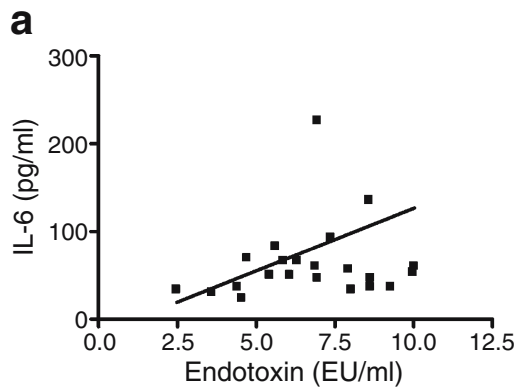

b

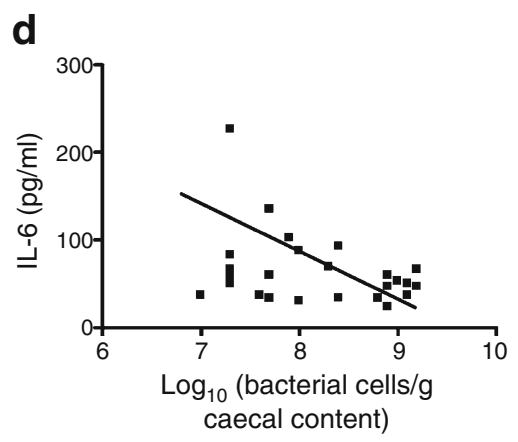

Fig. 7 Plasma markers of inflammation correlate positively with endotoxaemia and negatively with Bifidobacterium spp. Correlations between endotoxaemia and a IL-6, b IL-1 $\beta$ and c IL-1 $\alpha$. d Correlations between Bifidobacterium spp. and IL-6, e IL-1 $\beta$ and f
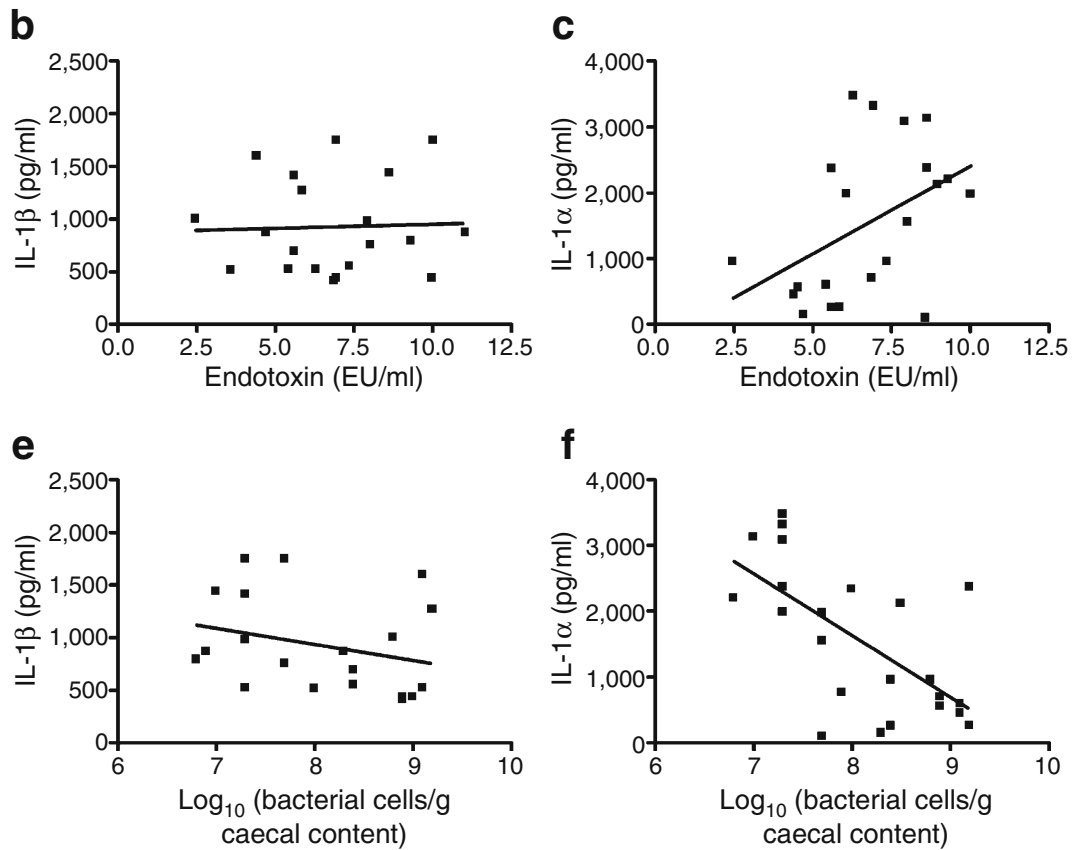

(b); $r=0.45$. Pearson's $r$ correlations: $r=0.3, p=0.16$ (a); $r=0.21, p=0.87$ (e); $r=-0.65, p=0.0008$ (f) 
the gut barrier function [34], i.e. in vitro butyrate, acetate and propionate all exert a concentration-dependent reduction of paracellular permeability in the Caco-2 model of colonic epithelium [35]. In the present study, we observed that HF-OFS-fed mice exhibit a significant increase in total and empty caecum weight compared with HF and HF-Cell mice (data not shown). This supports the idea that the fermentation of dietary fibre allowing the proliferation of specific bacteria such as bifidobacteria influences the profile of short-chain fatty acids released in the gut. The consequences of the specific fermentation are lower endotoxaemia and an improvement of metabolic disturbances.

With regard to the role of gut bacteria in the development of metabolic disorders, Brugman et al. have shown that antibiotic treatment partially protects against the occurrence of diabetes in a diabetes-prone rat that develops insulitis. The authors proposed that altering the gut microbiota composition by antibiotic treatment reduces the antigenic load and hence the inflammatory reaction that had led to pancreatic beta cell destruction [36]. Consistent with these results, we have recently demonstrated that HFdiet-fed mice and $o b / o b$ mice treated with antibiotics for 4 weeks were partially protected against the development of metabolic diseases and inflammatory tone (P. D. Cani, N. M. Delzenne and R. Burcelin, unpublished data).

We cannot rule out the putative implication of another important mechanism to explain the improved glucose metabolism in prebiotic-fed mice. Indeed, we have previously reported that prebiotic dietary fibre improved diabetes and insulin sensitivity by a mechanism promoting synthesis and secretion of the incretin, GLP-1 [18, 28, 29]. Here, we confirmed that prebiotic dietary fibre only strongly increase colonic proglucagon mRNA content. Therefore, the present data suggest that there is a link between modulation of gut microbiota, endotoxaemia, inflammation and GLP-1 secretion. This merits further investigation. While the doses used in animal studies are not directly transposable to human nutrition, several reports have demonstrated that ingestion of fructo-oligosaccharides at the dose of 5 to $20 \mathrm{~g} /$ day are sufficient to promote prebiotic [37-40] and physiological effects [41-44].

In conclusion, dietary modulation of gut microbiota with a view to increasing bifidobacteria reduced endotoxaemia and improved glucose tolerance and insulin secretion, as well as reducing inflammation development in HF-diet-fed mice. Together, these findings suggest that the gut microbiota contribute to the pathophysiological regulation of endotoxaemia and set the tone of inflammation, glucose tolerance and insulin secretion.

Thus, specific strategies for modifying gut microbiota in favour of bifidobacteria could be useful tools for reducing the impact of high-fat feeding on the occurrence of metabolic diseases.
Acknowledgements This work was supported by an FSR (Fonds Spéciaux de Recherche) grant from the Université catholique de Louvain and by a grant from the Fonds National de la Recherche Scientifique (FNRS 1.5.231.06-Belgium). P. D. Cani is a postdoctoral researcher from the FNRS Belgium. We would like to thank C. Feyt, and E. Delmée for helpful criticism and F. Debacker for excellent technical assistance.

Duality of interest The authors declare that there is no duality of interest associated with this manuscript.

\section{References}

1. Wellen KE, Hotamisligil GS (2005) Inflammation, stress, and diabetes. J Clin Invest 115:1111-1119

2. Kahn BB, Flier JS (2000) Obesity and insulin resistance. J Clin Invest 106:473-481

3. Hotamisligil GS, Shargill NS, Spiegelman BM (1993) Adipose expression of tumor necrosis factor-alpha: direct role in obesitylinked insulin resistance. Science 259:87-91

4. Weisberg SP, McCann D, Desai M, Rosenbaum M, Leibel RL, Ferrante AW, Jr (2003) Obesity is associated with macrophage accumulation in adipose tissue. J Clin Invest 112:1796-1808

5. Hotamisligil GS, Peraldi P, Budavari A, Ellis R, White MF, Spiegelman BM (1996) IRS-1-mediated inhibition of insulin receptor tyrosine kinase activity in TNF-alpha- and obesityinduced insulin resistance. Science 271:665-668

6. Plomgaard P, Bouzakri K, Krogh-Madsen R, Mittendorfer B, Zierath JR, Pedersen BK (2005) Tumor necrosis factor-alpha induces skeletal muscle insulin resistance in healthy human subjects via inhibition of Akt substrate 160 phosphorylation. Diabetes 54:2939-2945

7. Backhed F, Ding H, Wang T et al (2004) The gut microbiota as an environmental factor that regulates fat storage. Proc Natl Acad Sci USA 101:15718-15723

8. Backhed F, Ley RE, Sonnenburg JL, Peterson DA, Gordon JI (2005) Host-bacterial mutualism in the human intestine. Science 307:1915-1920

9. Backhed F, Manchester JK, Semenkovich CF, Gordon JI (2007) Mechanisms underlying the resistance to diet-induced obesity in germ-free mice. Proc Natl Acad Sci USA 104:979-984

10. Ley RE, Backhed F, Turnbaugh P, Lozupone CA, Knight RD, Gordon JI (2005) Obesity alters gut microbial ecology. Proc Natl Acad Sci USA 102:11070-11075

11. Ley RE, Turnbaugh PJ, Klein S, Gordon JI (2006) Microbial ecology: human gut microbes associated with obesity. Nature 444:10221023

12. Cani PD, Amar J, Iglesias MA et al (2007) Metabolic endotoxemia initiates obesity and insulin resistance. Diabetes 56:1761-1772

13. Griffiths EA, Duffy LC, Schanbacher FL et al (2004) In vivo effects of bifidobacteria and lactoferrin on gut endotoxin concentration and mucosal immunity in Balb/c mice. Dig Dis Sci 49:579-589

14. Wang Z, Xiao G, Yao Y, Guo S, Lu K, Sheng Z (2006) The role of bifidobacteria in gut barrier function after thermal injury in rats. $\mathrm{J}$ Trauma 61:650-657

15. Wang ZT, Yao YM, Xiao GX, Sheng ZY (2004) Risk factors of development of gut-derived bacterial translocation in thermally injured rats. World J Gastroenterol 10:1619-1624

16. Tuohy KM, Rouzaud GC, Bruck WM, Gibson GR (2005) Modulation of the human gut microflora towards improved health using prebiotics - assessment of efficacy. Curr Pharm Des 11:7590 
17. Cani PD, Dewever C, Delzenne NM (2004) Inulin-type fructans modulate gastrointestinal peptides involved in appetite regulation (glucagon-like peptide-1 and ghrelin) in rats. Br J Nutr 92:521-526

18. Cani PD, Knauf C, Iglesias MA, Drucker DJ, Delzenne NM, Burcelin R (2006) Improvement of glucose tolerance and hepatic insulin sensitivity by oligofructose requires a functional glucagonlike peptide 1 receptor. Diabetes 55:1484-1490

19. Franks AH, Harmsen HJ, Raangs GC, Jansen GJ, Schut F, Welling GW (1998) Variations of bacterial populations in human feces measured by fluorescent in situ hybridization with groupspecific 16S rRNA-targeted oligonucleotide probes. Appl Environ Microbiol 64:3336-3345

20. Harmsen HJ, Wildeboer-Veloo AC, Raangs GC et al (2000) Analysis of intestinal flora development in breast-fed and formula-fed infants by using molecular identification and detection methods. J Pediatr Gastroenterol Nutr 30:61-67

21. Langendijk PS, Schut F, Jansen GJ et al (1995) Quantitative fluorescence in situ hybridization of Bifidobacterium spp. with genus-specific 16S rRNA-targeted probes and its application in fecal samples. Appl Environ Microbiol 61:3069-3075

22. Manz W, Amann R, Ludwig W, Vancanneyt M, Schleifer KH (1996) Application of a suite of 16S rRNA-specific oligonucleotide probes designed to investigate bacteria of the phylum cytophaga-flavobacter-bacteroides in the natural environment. Microbiology 142:1097-1106

23. Salzman NH, de Jong H, Paterson Y, Harmsen HJ, Welling GW, Bos NA (2002) Analysis of 16S libraries of mouse gastrointestinal microflora reveals a large new group of mouse intestinal bacteria. Microbiology 148:3651-3660

24. Devereux R, Mundfrom GW (1994) A phylogenetic tree of 16S rRNA sequences from sulfate-reducing bacteria in a sandy marine sediment. Appl Environ Microbiol 60:3437-3439

25. Ootsubo M, Shimizu T, Tanaka R et al (2002) Oligonucleotide probe for detecting Enterobacteriaceae by in situ hybridization. J Appl Microbiol 93:60-68

26. Knauf C, Rieusset J, Foretz M et al (2006) Peroxisome proliferator-activated receptor-alpha-null mice have increased white adipose tissue glucose utilization, GLUT4, and fat mass: role in liver and brain. Endocrinology 147:4067-4078

27. Cani PD, Neyrinck AM, Maton N, Delzenne NM (2005) Oligofructose promotes satiety in rats fed a high-fat diet: involvement of glucagon-like peptide-1. Obes Res 13:1000-1007

28. Cani PD, Daubioul CA, Reusens B, Remacle C, Catillon G, Delzenne NM (2005) Involvement of endogenous glucagon-like peptide-1(7-36) amide on glycaemia-lowering effect of oligofructose in streptozotocin-treated rats. J Endocrinol 185:457-465

29. Cani PD, Hoste S, Guiot Y, Delzenne NM (2007) Dietary nondigestible carbohydrates promote L-cell differentiation in the proximal colon of rats. Br J Nutr 98:32-37
30. Brun P, Castagliuolo I, Leo VD et al (2007) Increased intestinal permeability in obese mice: new evidence in the pathogenesis of nonalcoholic steatohepatitis. Am J Physiol Gastrointest Liver Physiol 292:G518-G525

31. Caplan MS, Miller-Catchpole R, Kaup S et al (1999) Bifidobacterial supplementation reduces the incidence of necrotizing enterocolitis in a neonatal rat model. Gastroenterology 117:577-583

32. Commane DM, Shortt CT, Silvi S, Cresci A, Hughes RM, Rowland IR (2005) Effects of fermentation products of pro- and prebiotics on trans-epithelial electrical resistance in an in vitro model of the colon. Nutr Cancer 51:102-109

33. Spindler-Vesel A, Bengmark S, Vovk I, Cerovic O, Kompan L (2007) Synbiotics, prebiotics, glutamine, or peptide in early enteral nutrition: a randomized study in trauma patients. JPEN J Parenter Enteral Nutr 31:119-126

34. Guarner F (2005) Inulin and oligofructose: impact on intestinal diseases and disorders. Br J Nutr 93(Suppl 1):S61-S65

35. Mariadason JM, Barkla DH, Gibson PR (1997) Effect of shortchain fatty acids on paracellular permeability in Caco-2 intestinal epithelium model. Am J Physiol 272:G705-G712

36. Brugman S, Klatter FA, Visser JT et al (2006) Antibiotic treatment partially protects against type 1 diabetes in the Bio-Breeding diabetes-prone rat. Is the gut flora involved in the development of type 1 diabetes? Diabetologia 49:2105-2108

37. Gibson GR, Willems A, Reading S, Collins MD (1996) Fermentation of non-digestible oligosaccharides by human colonic bacteria. Proc Nutr Soc 55:899-912

38. Gibson GR (1998) Dietary modulation of the human gut microflora using prebiotics. Br J Nutr 80:S209-S212

39. Gibson GR (1999) Dietary modulation of the human gut microflora using the prebiotics oligofructose and inulin. J Nutr 129:1438S-1441S

40. Gibson GR, Roberfroid MB (1995) Dietary modulation of the human colonic microbiota: introducing the concept of prebiotics. J Nutr 125:1401-1412

41. Scholz-Ahrens KE, Ade P, Marten B et al (2007) Prebiotics, probiotics, and synbiotics affect mineral absorption, bone mineral content, and bone structure. J Nutr 137:838S-846S

42. Cani PD, Joly E, Horsmans Y, Delzenne NM (2006) Oligofructose promotes satiety in healthy human: a pilot study. Eur J Clin Nutr 60:567-572

43. Daubioul CA, Horsmans Y, Lambert P, Danse E, Delzenne NM (2005) Effects of oligofructose on glucose and lipid metabolism in patients with nonalcoholic steatohepatitis: results of a pilot study. Eur J Clin Nutr 59:723-726

44. Letexier D, Diraison F, Beylot M (2003) Addition of inulin to a moderately high-carbohydrate diet reduces hepatic lipogenesis and plasma triacylglycerol concentrations in humans. Am J Clin Nutr 77:559-564 